\title{
AN APPROACH FOR THE ASCERTAINMENT OF PROFIT SHARES FOR NETWORK PARTICIPANTS
}

\author{
Hendrik Jähn, Marco Fischer, Matthias Zimmermann \\ Chemnitz University of Technology, hendrik.jaehn@wirtschaft.tu-chemnitz.de \\ GERMANY
}

\begin{abstract}
This paper introduces an approach for the automated calculation of profit shares for participants in non-hierarchical production networks under consideration of incentive and sanction mechanisms. Thereby the sanctions can be ascertained within an integrated approach for the evaluation of the performance of the individual value-adding units. Incentive mechanisms focus the interference of the behavior of the value-adding-units in order to act network-compliant. The discussed issues concern the two final phases of a typical life cycle of a virtual enterprise: the phase of the evaluation and the phase of the break-up of the network. Thereby the maximization of utility of the entire virtual enterprise serves as the main objective.
\end{abstract}

\section{INTRODUCTION}

The current economic situation is characterized by the requirement for a flexible and customer-oriented production of goods. As one reaction in order to bear that challenge, many enterprises intensify their engagement in virtual enterprises. At Chemnitz University of Technology, an innovative concept for the cooperation especially of very small organization units is elaborated within the scope of the Collaborative Research Centre 457 (Müller, 2003). That concept is called „Nonhierarchical Regional Production Networks“. The cooperating organization units thereby are called Competence Cells (CCs). For operating and coordinating that kind of collaborative networks the management concept "Extended Value Chain Management" (EVCM) was developed (Teich, 2003). It guarantees the adherence to the non-hierarchical structure. That means that all CCs are legally and economically independent and dispose of equal rights and opportunities. Therefore the selection of the partners as well as the planning and execution of the value-adding process take place as far as possible in an automated way coordinated by EVCM. Because of the assumption of an asymmetrical distribution of information within the network it is expected that every network participant will act in favour of its own maximization of utility (Macho-Stadler, 2001). However for the success of the entire network the maximization of the network utility must be aimed at. Therefore suitable incentive and sanction mechanisms for the CCs are necessary to manage the problems caused by these behavior patterns. 
In this contribution an approach for the automated calculation of profit shares in non-hierarchical regional Production Networks under consideration of incentive and sanction mechanisms is introduced. The consideration of incentives and sanctions within the calculation of profit shares for network participants describes an innovative approach. It has to be highlighted that the distribution of profit is a very important issue for the success of the entire network. However this problem is little discussed from the theoretical perspective. For that case only little literature exists (Rehkugler, 1972). The approach assumes that the CCs have well defined insulated tasks which is appropriate especially in the job-shop production in machine building.

\section{DISTRIBUTION OF PROFIT}

Although the topic of the distribution of profit only gains importance after finishing a value-adding process, the expectations of the CCs concerning profit already have to be taken into consideration during their selection phase. In the following, a model which focuses on the determination as well as the distribution of profits is introduced.

The basis of the model for a fair distribution of profit among partners in virtual enterprises is the phase of the preparation and submission of offers for every valueadding step. Therefore, a customer inquiry must have been made to the network for an exactly defined product. It has to be distinguished whether the customer expresses his idea of the price or not. In case, the customer states a desired purchase price, that price needs to be compared to the price that can be realized by the production network. That parameter can be calculated from the sum of the evaluated value-adding parts of all the CCs participating in the network plus a network profit, which is calculated as the sum of the profit expectations of the single CC. In case, the realizable price is higher than the price desired by the customer, it has to be expected that the customer does not accept the offer. In that case negotiations are necessary (Jähn, 2004). If the realizable price as oppose is lower than the desired price, the good is offered at the desired price for realizing a correspondingly higher network profit or to set up reserves.

In reverse, it is possible to ascertain a profit from the difference of the desired price and the realizable delivery price without profit. That profit amount can subsequently be distributed to the single CCs involved in the value-adding process by the means of a specific distribution key. After that, the CCs compare their individual idea of the profit and the planned profit share. If the majority accepts that amount, an offer can be submitted to the potential customer containing the desired price. The customer will probably accept that offer. In that case, the profit is calculated from the difference of the desired price and the realizable delivery price. The value-adding process can be started.

That procedure is more complicated in case several CCs are not satisfied with their profit share. This is similar to the case when the customer has no concrete idea concerning the desired price. In that case a new price must be ascertained. Here, the individual profit ideas of the single CCs serve as calculation basis. The profit ideas should be ascertained as a percentage of the value-adding share of a CC. An offer price for the inquired product can be calculated from the sum of the individual value-adding shares and the individual percentages of the profit shares. That price is reported to the potential customer. This customer has to decide if he accepts the 
offer or not. In case he accepts the offer, the profit is fixed and the production of the inquired good can be started. As oppose, if the customer cannot accept the offer because of the price, it has to be analyzed what can be modified. Thus, the customer might be convinced to pay a higher price. If this is not possible, the CCs need to reduce their profit ideas. Thereby, the question has to be answered whether a reduction is possible in order to realize the desired price of the customer. For the case that this is not possible and the customer is not willing to pay a higher price, the negotiation procedure is cancelled and no contract is concluded. That means the customer does not place the order. This should be an exception. In that case negotiations concerning the product can be started in order to realize an attractive offer, even the product differs from the original inquiry. Thereby it has to be paid attention that the requirements to the final product of the customer are fulfilled. In the next section the procedure concerning the distribution of profit is introduced.

Basically the total offer price is calculated from the sum of all the prices of the value-adding process of all $\mathrm{CCs}$ plus the expected profit shares per CC. After carrying through the value-adding process and delivering the final product to the customer, he is expected to pay the agreed purchase price. The agreed prices per value-adding process then are directly paid out to the participating CCs by EVCM. The remaining amount is the distributable profit $G$. It has to be mentioned that the distributable profit needs not to be equal to the expectations dependent on the procedure of the ascertainment of the offer price.

When ascertaining the individual profit share of a $\mathrm{CC}$, a differentiation of a fixed and a variable profit share in networks seems to be efficient for every $\mathrm{CC}$ for the basic model of the profit distribution in non-hierarchical regional production networks. For the ascertainment of that distribution parameter $\alpha$, it presents itself to compare the proportion of overheads and complete costs weighted with the realized value-adding $p_{i}$ of a $\mathrm{CC}$ to the complete value-adding of the production network. Equation 1 shows these interdependencies. $G$ represents the total amount of profit to be distributed.

$$
g_{i}=\alpha \cdot \frac{G}{n}+(1-\alpha) \cdot \frac{G \cdot p_{i}}{P_{\text {netto }}}
$$

If a finite number of CCs $n$ in the production network is assumed, which were involved in a certain value-adding process, a fixed profit share results which is equal for all participating CCs $i=1, \ldots, n$. As oppose, the variable part of a CC is calculated dependent on the individual share of value-adding $p_{i}$. Thus, this part is orientated on the corresponding offer price. Because a low offer price increases the probability of the participation in the network, it is avoided that single CCs might secure a too high share of the profit for themselves. The sum of the fixed and the variable profit share is the payable profit share $g_{i}$ of a CC. Both parts of the profit are weighted by $\alpha$.

That share $g_{i}$ has to be compared to the expected share from the offer submission. If the payable profit share $g_{i}$ is higher than the expected profit share $g_{i, e}$ of a $\mathrm{CC} i$, only the expected amount is paid out and the remaining amount is kept as a reserve for the EVCM. However, if the payable profit share is lower than the expected profit share of a $\mathrm{CC}$, an additional payment needs to be disposed that is financed by the reserves. Nevertheless, this must remain an exceptional case. 


\section{INCENTIVES}

Incentive mechanisms are mainly (but not exclusively) financial benefits that are paid by an employer to a contractor. Thereby, the employer tries to influence the behavior of the contractor to convince him to act in his interest. The reason why the contractor does not automatically act in his sense could be that each actor aims at maximizing his own utility. In case the client and the contractor do not pursue the same or at least similar aims, incentives have to be given in order to synchronize their utility. The choice of the adequate amount of the financial incentive thereby is very important. If one succeeds to financially quantify the utility difference, the incentive amount should be at least as high as that utility difference. However, it is more efficient that the incentive amount is a little higher than the utility difference for giving the contractor an incentive to decide in favour of the aims of the client.

Reasons for that have to be identified before incentives can be quantified. Thus, however, if a $\mathrm{CC}$ abandons the participation in a value-adding process, it can be the best alternative for it, but not necessarily for the entire network. This would for example be the case if a competence or $\mathrm{CC}$ is missing in a value-adding chain for being able to submit an offer. In that case, the missing $\mathrm{CC}$ has to be given suitable incentives for the participation in the network. Because a too low price is no reason for not participating in the network, other reasons must be found. Those could for example be lacking production capacities, lacking personnel, a lack of interest in participating in the network or not meeting the profit expectations. Two reasons are exemplarily explained in the next section. In these cases, the additional costs for a rescheduling of the involved $\mathrm{CC}$ or a simple monetary incentive for extending the profit need to be compensated. This is the content of the following model.

When taking the case into consideration, that one or more CCs are not able or willing to participate in the network, they have to be convinced by the help of certain incentive mechanisms to participate in the value added chain. Because of the assumption that every enterprise works for maximizing its profit, a (monetary) incentive is the logical consequence. This however presupposes that the CCs that are willing to participate in the network from the beginning on have to pay for that. However, the complete network profit needs to be ascertained for finding out about the exact amount of possible incentive payments which every $\mathrm{CC}$ has to make. The costs for the incentive payments have to be financed from that amount. As can be seen from the profit distribution model, an acclamation procedure is necessary for the ascertainment of the profit $G$. For the CC lacking in the value-adding network, it presents itself to introduce so-called CCsim (simulated $C C$ ) for being able to submit an offer for the potential customer and to ascertain the profit. Thereby, however, the number of the CCsim must not be bigger than the total number of CCs cooperating in the network minus 1.

For the calculation, it is now assumed that the simulated CCs fulfill all the requirements (with regard to capacities, personnel, soft-facts etc.) and in the acclamations agree to the majority of the CCs concerning $G$. Thus, it is possible to ascertain the theoretically achievable network profit $G_{t}$. For realizing that profit, however, it is necessary to find suitable CCs also for the vacant value-adding processes. The following two cases can be distinguished in the selection of those CCs: In the first case the CC, which was replaced by a CCsim, exists in the pool of resources and disposes of all the necessary resources, but however does not want to 
participate in the network under the given conditions. In the second case the $\mathrm{CC}$, which was replaced by a CCsim, does not exist in the network and / or does not dispose of all the necessary resources. There is the possibility that the CC-related share of value-adding $p_{i}$ is published by the potential $\mathrm{CC}$ which is absolutely necessary for the further calculation. However, if there is no exact number for $p_{i}$, either experiences or estimations must serve for the first step of the calculation.

As mentioned above, the already engaged CCs have to pay for the incentives which are granted. However, it has to be assumed that those CCs also have ideas concerning their individual profit share. If they know that no network can be formed without the missing $\mathrm{CC}$, they will nevertheless be willing to make the compensation payments. The reason results from the fact that no profit would be realizable at all without a network and thus a lower utility level would be achieved. Another acclamation procedure is carried through for the determination of the individual shares of the compensation payments (incentives). Within the scope of that process, the $\mathrm{CC}$ state to which amount (percentage of the complete result) they are ready to dispense with their profit. Equation (2) illustrates that coherence.

$$
i_{i}=\frac{p_{i}}{\sum_{i=1}^{n} p_{i}} \cdot p_{i} \cdot i_{R P i}
$$

Thereby $i_{i}$ refers to the individual profit share and $i_{R P i}$ the share a $\mathrm{CC}$ is willing to emit of its planned profit share in percent.

Subsequently, the amount of the available compensation payments $I$ can be ascertained. That corresponds to the average compensation share a CC is willing to use for incentive payments. This is illustrated by Equation (3).

$$
I=\sum_{i=1}^{n} i_{i}
$$

Now, the single incentive payments can be distributed individually to the not yet participating $\mathrm{CC}$, corresponding to their missing resources. The expenses for making available the resources have to be quantified for that. It thereby has to be considered that the $\mathrm{CC}$ won for the network also have to be involved in the compensation payment. After the available incentive budget $I$ has been ascertained, it is now possible to replace the CCsim by real CCs, thereby the aforementioned cases are distinguished again. For the distribution of the compensation payments to the new $\mathrm{CC}$, one starts with those from the second case. Those usually represent payments which are to make available the lacking resources and thus are only a little negotiable. The remaining amount of the available incentive budget $I$ is proportionately distributed to the $\mathrm{CC}$ which belong to the first case. If the required costs are higher than the available compensation payments $I$, the already participating CC are again asked to state the profit share they are ready to dispense with. The condition for that is that this is higher than in the first acclamation. Subsequently, the compensation payments are distributed again aiming at finding missing network partners. This procedure can now be repeated as often as necessary until the required CCs have been found. However, the process is broken up if the sum of the compensation payments gets bigger than the achievable profit or if one or 
more of the existing $\mathrm{CCs}$ leave the network because of too low expected profits. This would again result in additional costs.

Incentive payments are based on the assumption that all the CCs aim at an individual maximization of utility. Thus, it must always be desirable to participate in a value-adding process if a profit is made. However, if a customer order cannot be realized because of lacking CCs, a profit of 0 would be achieved which is not desirable. Therefore, compensation payments as incentives are justified.

After introducing the model for distributing profit and the consideration of incentives a quantitatively orientated model for the calculation of sanctions for CCs, that have not performed a service as it was planned, is introduced in the next section. That model is based on a basic performance evaluation of each $\mathrm{CC}$ involved in the value-adding process.

\section{SANCTIONS}

If the evaluation of the performances of the network participants is aimed, the most important influence parameters need to be identified in a first and singular or at the most periodical work step. They include the price, the date of delivery and the specification of the quality. The quantity however is assumed as a fixed parameter and thus it is not included in the analysis. On the other hand, relevant parameters result from the social connection of such networked structures. Those for example include the quality of the cooperation and the confidential climate among the network participants. Thus, it is valid that there are $m$ parameters $K_{j}, j=1, \ldots, m(m=5)$ to be investigated.

In order to manage the different relevancy of the single parameters, a weighting $l_{j}$ has to be determined for every parameter in the second phase. The determination of the weights is based on the controlling target system. This is also a singular or periodical task. Rankings or empirical investigations might be resources for that. In order to achieve a possibility of comparison of the parameters in order to standardize the evaluation, in the regular case it is valid: $\Sigma l_{j}=L=1$. Thus, it is guaranteed that the parameters can be compared with regard to their significance.

In order to be able to carry through a target-actual value-comparison of the parameters, the maximally achievable evaluation variable $t v$ (target value) must be ascertained for every parameter $j$ in phase three. This evaluation variable then must be compared to the actually achieved evaluation variable $a v$ (actual value) of every parameter $j$ and of every $\mathrm{CC} i$ after each value-adding process in a dynamic network. That maximally achievable evaluation variable results from the product of the maximally achievable number of credits $r_{j, \max }$ (usually 10) and the weight $l_{j}$, cf. equation (4).

$$
t v_{j}=l_{j} \cdot r_{j, \max }
$$

For managing that task, the degree of fulfillment, that justifies a maximal number of credits, must be fixed for every parameter involved. That task has a different difficulty for the single parameters. While keeping the price agreed upon and sticking to the determined quality justify an obviously maximal credit evaluation, the evaluation of the soft-facts is far more difficult. Therefore, an approach would be the evaluation with the maximal number of credits in case of an 
"optimal“ confidential culture and an "optimal“ quality of the cooperation. Nevertheless, this issue remains subjective to a large extent. Most practicable for the evaluation of soft-facts in short terms is the individual rating of the parameters by the enterprises. However in this case data can be gerrymandered. The summed up target value $(T V)$ can be ascertained by adding all $t v_{j}$ for all considered parameters $K_{j}$. This value of comparison value is valid for several value-adding processes.

The following working steps need to be carried through for every process of value-adding and therefore they provide highly dynamic data for evaluating the performance of the enterprises during value-adding.

At first the five in the previous paragraph identified parameters are evaluated by the means of suitable points and evaluation measures with regard to their degrees of fulfillment for every CC. That credit evaluation is called $r_{i j}$ and normally amounts to a value between 0 (extremely bad performance) and 10 (excellent / perfect performance). A connection between the degree of fulfillment and the credit evaluation needs to be fixed for every parameter. For considering the performance of the single CCs during the value-adding process the actual value is calculated. By multiplying the achieved credits $r_{i j}$ and the corresponding weighting $l_{j}$, a weighted credit, the so-called actual value $a v_{i j}$, results for every parameter and every $\mathrm{CC}$, therefore cf. equation (5).

$$
a v_{i j}=l_{j} \cdot r_{i j}
$$

Subsequently, the aggregated actual value of a CC $i\left(A V_{i}\right)$ can be ascertained by summing up the $a v_{i j}$ for all the parameters $K_{j}$. Then, that value serves as a basis for the further analysis. $A V_{i}$ describes in one variable the performance of an enterprise without giving any information about the quality of the performance.

Therefore finally the measure $N C_{i}$ (network conformity) has to be ascertained in phase five by the comparison of the actual value $A V_{i}$ and the target value $T V$ for the evaluation of the behavior of a $\mathrm{CC}$ in the virtual enterprise. The network conformity represents a kind of level of fulfillment of the completed performances, because in the calculation, the aggregated actual value is compared to the aggregated target value. Thus, this variable can also be interpreted as the percentage of the fulfillment of performances. The calculation rule for that is illustrated in equation (6).

$$
N C_{i}=\frac{A V_{i}}{T V}
$$

The variable $N C$ represents the degree of the network conformity of a $\mathrm{CC}$ and concludes in only one value the quality of fulfillment of the several performance parameters of the enterprises participating in a special dynamic network. That parameter is re-ascertained for every value-adding process for every participating $\mathrm{CC}$ according to the pattern described above. The comparability with other CCs as well as the consideration for the distribution of profit thus is guaranteed.

Finally, the $N C_{i}$ of a $\mathrm{CC}$ must be allocated to the application in the profit distribution model within the scope of sanction mechanisms. In case of a poor performance of a $\mathrm{CC}$, a proportionate shortage of the profit $s_{i}$ seems to be an efficient measure. The sanction amount can be calculated according to equation (7).

$$
s_{i}=\left(1-N C_{i}\right) \cdot g_{i}
$$

The remaining share of the profit has to be paid to the enterprises involved in the value-adding process in the network after the customer has paid the amount of an 
invoice. In case a $\mathrm{CC}$ has performed very well and has kept its delivery promises fixed in the contract it will receive the full profit share. An outstanding performance of the entire network is aimed at by allying this approach within the network controlling.

\section{AGGREGATION}

After the quantification of incentive and sanction payments, the final profit share $z_{i}$ for every CC $i$ can be calculated. That amount is finally to be paid to the CCs. Thus, after finishing a value-adding process, a CC is on the one hand given the individual share of the value-adding $p_{i}$ and on the other hand the corresponding profit $g_{i}$. In addition, the incentives $i_{i}$ are paid out and possible sanctions $s_{i}$ are subtracted. It is stressed here that $i_{i}$ as well as $s_{i}$ might consist of several components. By realizing just one money flow including all components a clear calculation procedure realized by EVCM can be guaranteed. It has to be stressed that the calculation of profit shares is a very complex issue and the acceptance of the calculation scheme of all affected CCs is precondition for its implementation.

\section{CONCLUSION}

This paper introduced an approach for the profit distribution in non-hierarchical regional production networks taking into consideration sanction and incentive mechanisms. The described methodology is a starting point for dealing with a key issue in collaboration among independent partners. However further development will certainly be needed. This includes the procedure of evaluation and rating and the necessary instance for fulfilling that task. Furthermore a disturbance management has to be included in case a partner does not accept the ratings or sanctions. It has also to be clarified that monetary incentives are not the only ones.

However, the introduced approach can be arbitrarily expanded and adapted to certain structures within specific virtual enterprises. Future work will focus on the refinement of that approach as well as on its information-technical implementation in the EVCM. The realization of the approach makes possible a contribution to an automated and efficient network controlling and hereby supports the success of virtual enterprises in a rapidly changing world-wide economic environment.

\section{REFERENCES}

1. Jähn H, Zschorn L, Zimmermann M. An automated Negotiation Model for non-hierarchical Production Networks. In Proceedings of the $8^{\text {th }}$ International Conference on Manufacturing Management, Vol.1, Salem S, ed, Gold Coast, Queensland, Australia, December 08-10, pp. 125-132, 2004.

2. Macho-Stadler I, Pérez-Castrillo, JD. An Introduction to the Economics of Information: Incentives and Contracts, Second Edition, Oxford: Oxford University Press, 2001.

3. Müller E, Wirth S. Innovative Production Nets based on a Competence Cell-based Approach. In Current Trends in Production Management, Zülch G et al., eds, 268-274, Aachen: Shaker, 2003.

4. Rehkugler, H. Die Verteilung einzelwirtschaftlicher Wertschöpfung. Dissertationsschrift, LudwigMaximilians-Universität zu München, 1972.

5. Teich T. Extended Value Chain Management (EVCM): Ein Konzept zur Koordination von Wertschöpfungsnetzen, Chemnitz: Verlag der GUC, 2003. 